\title{
Organic matter in the Solar System: From colors to spectral bands
}

\author{
Dale P. Cruikshank
}

NASA Ames Research Center

MS 245-6

Moffett Field, CA 94035 USA

email: Dale.P.Cruikshank@nasa.gov

\begin{abstract}
The reflected spectral energy distribution of low-albedo, red-colored, airless bodies in the outer Solar System (planetary satellites, Centaur objects, Kuiper Belt objects, bare comet nuclei) can be modeled with spectral models that incorporate the optical properties of refractory complex organic materials synthesized in the laboratory and called tholins. These materials are strongly colored and impart their color properties to the models. The colors of the bodies cannot be matched with plausible minerals, ices, or metals. Iapetus, a satellite of Saturn, is one such redcolored body that is well matched with tholin-rich models. Detection of aromatic and aliphatic hydrocarbons on Iapetus by the Cassini spacecraft, and the presence of these hydrocarbons in the tholins, is taken as evidence for the widespread presence of solid organic complexes aromatic and aliphatic units on many bodies in the outer Solar System. These organic complexes may be compositionally similar to the insoluble organic matter in some classes of the carbonaceous meteorites, and thus may ultimately derive from the organic matter in the interstellar medium.
\end{abstract}

Keywords. Astrochemistry, Planets and satellites, Kuiper Belt, Molecules

\section{Introduction}

Small bodies in the outer Solar System (planetary satellites, comet nuclei, Kuiper Belt objects, and Centaurs) are highly diverse in terms of orbital characteristics, dimensions, surface structures, and spectral characteristics. Many small bodies are thought to be remnant planetesimals or collisional fragments of planetesimals, preserved largely intact since their condensation in the solar nebula. As such, to the degree that they are chemically unaltered, they represent primordial material that can offer clues to the composition of the Solar System's nascent molecular cloud and the processes that occurred within the solar nebula during the epoch of planet formation. In addition to rocky material, metals, and ices, it is clear that organic molecular material is also often present. This organic component must be identified and understood as we progress toward a complete view of the original chemistry and processes in the early Solar System that led to the compositions we now see, and to the origin of life, at least on one planet.

This paper discusses new observations and insight into the presence of complex organic macromolecular material as a component of the low albedo surfaces that are typically found on small planetary satellites, the nuclei of comets, and on members of the newly identified populations of bodies beyond the orbit of Neptune.

These objects are typically too distant and faint for the application of infrared spectroscopy in the spectral regions where organic molecules have characteristic spectral bands $(\lambda>3 \mu \mathrm{m})$. They can, however, be observed photometrically over a sufficient wavelength interval to determine their colors, typically in a portion of the spectral region in which diffusely reflected sunlight dominates the spectral energy distribution $(\lambda<5 \mu \mathrm{m})$. 
For very faint objects the observable spectral interval includes $\sim 0.3-1 \mu \mathrm{m}$, while for brighter objects the range can be extended to $2.5 \mu \mathrm{m}$ and beyond. Photometry of large samples of the asteroids shows a wide variation in color, from neutral (or slightly blue) with respect to the Sun, to very red (Chapman et al. 1975, Zellner et al. 1985). These observational studies extended to small planetary satellites (Degewij et al. 1980), comets (Hartmann et al. 1982), and Kuiper Belt objects (Barucci et al. 2001, 2004) revealed similar trends, with a preponderance of red objects in these populations. At about the same time, parallel observations of the thermal emission of many of these objects at longer infrared wavelengths (typically 10 and $20 \mu \mathrm{m}$; e.g., Morrison 1973, Cruikshank \& Morrison 1974) revealed the overall very low albedos ( $<0.08$ at the V-band wavelength) in the majority of cases.

\section{Organic Solids and the Surface Colors of Solar System Bodies}

In an effort to understand the compositions of the reddest asteroids (the D class), Gradie and Veverka (1980) introduced the concept of complex organic solid materials on their surfaces, suggesting the presence of '... very red, very opaque, polymer-type organic compounds, which are structurally similar to aromatic-type kerogen...' Laboratory work by Sagan and Khare (1979) in progress at Cornell University, Gradie and Veverka's home institution, was at that time showing the importance and possible relevance of such organic compounds in explaining certain features of interstellar dust and the aerosol component of Titan's atmosphere. This synthetic organic solid, showing strong colors ranging from yellow through red to black, was termed 'tholin' by Sagan.

The optical properties (complex refractive indices) measured for several tholins (e.g., Khare et al. 1984, MacDonald et al. 1994) have enabled the use of these materials in calculations of synthetic spectra of diffusely scattered sunlight from a particulate solid surface for comparison with the observational data (e.g., Cruikshank \& Dalle Ore 2003, Barucci et al. 2005). In the case of the extremely red Centaur object, 5145 Pholus, and by extension to other red bodies, it was demonstrated that the complex organic solid material (tholin) is an essential ingredient of the models to produce the observed color (Cruikshank et al. 1998). Recent work on the synthesis and analysis of tholins, primarily in the context of Titan's aerosol, is reported by Imanaka et al. (2004) and Bernard et al. (2006). The importance of tholins as coloring agents on bodies in the outer Solar System is discussed in detail by Cruikshank et al. (2005).

While minerals and ices do not give satisfactory spectral fits to the strongly redcolored surfaces of Solar System bodies, whereas models containing tholins do, it has been assumed for many years that organic solids are present on those surfaces, even though specific and diagnostic spectral absorption bands could not be detected. With the in situ study of the satellites of Saturn with VIMS (Visible-Infrared Mapping Spectrometer) aboard the Cassini spacecraft, that situation has changed with the detection of aromatic and aliphatic hydrocarbon bands in the red-colored, low-albedo material on Iapetus, Phoebe, and Hyperion (Clark et al. 2005, Cruikshank et al. 2008).

\section{Results from the Satellites of Saturn}

Iapetus. In this paper we focus on the surface compositions of the reddest Solar System bodies, using the measurements of three of Saturn's satellites, most specifically Iapetus, obtained by the VIMS instrument on the Cassini spacecraft, and then generalize to other surfaces whose spectra can be satisfactorily modeled by the use of tholins as the principal coloring agent. 
Iapetus has the unique property among planetary satellites that the hemisphere centered on the apex of its locked synchronous orbital motion around Saturn has a geometric albedo of $0.02-0.06$, while most of the remainder of the surface is some ten times more reflective and shows characteristic spectral absorption bands of $\mathrm{H}_{2} \mathrm{O}$ ice. The mean density of Iapetus is $1.1 \mathrm{~g} \mathrm{~cm}^{-3}$, suggestive of a bulk composition dominated by $\mathrm{H}_{2} \mathrm{O}$ ice, but note that organic macromolecular solids also have a similar density. The pattern of distribution of the ice and the low-albedo material is seen to be more complex in the detailed images obtained by Cassini in December 2004 and in September 2007 than that deduced from ground-based data and images from Voyagers 1 and 2, but the dominance of an almost uninterrupted low albedo surface centered on the leading hemisphere

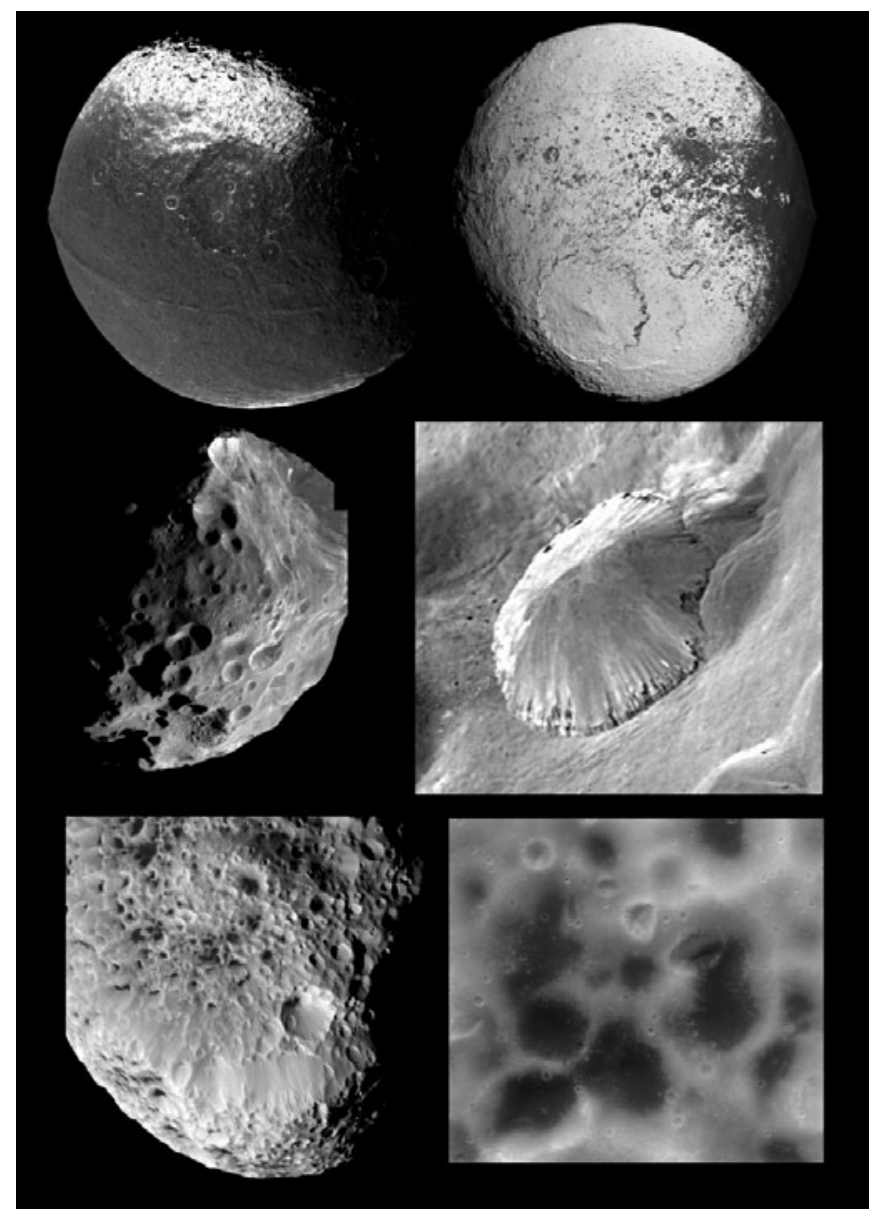

Figure 1. Three satellites of Saturn for which near-infrared reflectance spectra reveal aromatic and aliphatic hydrocarbon bands. Top two images show two different hemispheres of Iapetus (mean diameter $1470 \mathrm{~km}$ ); the low-albedo material on the left image is distributed around the apex of the satellites locked-synchronous revolution around Saturn. The hydrocarbon bands occur mainly in the low-albedo material. The bright material is water ice. The central two images show Phoebe (long dimension $230 \mathrm{~km}$ ). The hydrocarbon bands are distributed non-uniformly across the surface, which also shows water ice absorption bands. Center right image shows an apparent shallow subsurface layer of especially low-albedo material in a crater rim (crater named Euphemus, diameter about $40 \mathrm{~km}$ ). Bottom left is a portion of Hyperion (dimensions $360 \mathrm{x} 280$ x $225 \mathrm{~km}$ ) showing a largely water-ice surface with low-albedo material concentrated in some crater interiors (enlarged on bottom right; crater diameters about $6 \mathrm{~km}$ ). NASA images from the Cassini spacecraft. 
remains a unique and intriguing feature of this satellite. Because no other known object shows such a global albedo contrast, an explanation of its cause on Iapetus must also address the absence of such a brightness distribution on any of the other satellites of the major planets (Buratti et al. 2005) or transneptunian bodies known to date.

The overall spectral reflectance of the low-albedo material on Iapetus shows a very red color, a weak absorption band at $0.67 \mu \mathrm{m}$, and a strong absorption band at $3 \mu \mathrm{m}$ [see Buratti et al. (2005) and Owen et al. (2001) for details and references]. Owen et al. (2001) modeled the low-albedo material with an intimate mixture of (nitrogenrich) tholin, amorphous carbon, and $\mathrm{H}_{2} \mathrm{O}$ ice. In their models the tholin provided the red color and a strong absorption band (N-H stretching mode) at $3 \mu \mathrm{m}$, matching the albedo, overall spectral shape, and the profile of the $3-\mu \mathrm{m}$ band throughout the spectral region 0.3-3.8 $\mu \mathrm{m}$.

The first complete coverage of the spectrum of Iapetus from $0.4-5.1 \mu \mathrm{m}$, without the interruptions induced by the Earth's atmosphere, was afforded by the VIMS instrument (Brown et al. 2004) on the Cassini spacecraft in December, 2004. Weak residual $\mathrm{H}_{2} \mathrm{O}$ ice absorption bands and the $4.27 \mu \mathrm{m} \mathrm{CO}_{2}$ band were clearly seen (Buratti et al. 2005, Brown et al. 2006). Initial models of the Cassini VIMS spectrum presented in Buratti et al. (2005) use tholins as the principal coloring agent, and also include HCN polymer, which acts to lower the albedo in the model to match that of Iapetus closely. Further detailed analysis of the 3.0-3.7 $\mu \mathrm{m}$ spectral region yielded the detection of the $3.3-\mu \mathrm{m}$ $\mathrm{C}-\mathrm{H}$ stretching mode band in aromatic hydrocarbons and components of the 3.4- $\mu \mathrm{m}$ aliphatic C-H stretching mode band complex (Cruikshank et al. 2008). This detection is corroborated and strengthened in additional spectra of the low-albedo surface units obtained at another close flyby of Iapetus in September, 2007.

Phoebe and Hyperion. The VIMS data for two other satellites of Saturn also give indications of the aromatic and aliphatic C-H bands (Clark et al. 2005, Cruikshank et al. 2007, Coradini et al. 2008), also distributed nonuniformly, but the signatures are weaker than on Iapetus, and a detailed analysis has not yet been completed. Other common characteristics of the low-albedo material on Iapetus, Phoebe, and Hyperion include the presence of $\mathrm{CO}_{2}$ absorption band at $4.27 \mu \mathrm{m}$, a prominent but unidentified absorption band at $2.42 \mu \mathrm{m}$, and residual $\mathrm{H}_{2} \mathrm{O}$ ice absorption. The band attributed to $\mathrm{CO}_{2}$ has not been fully elucidated because of shifts in the band's central wavelength outside the range expected for pure $\mathrm{CO}_{2}$ ice (Cruikshank et al. 2007, Clark et al. 2008). This matter is currently under study.

\section{Discussion}

The aromatic and aliphatic hydrocarbon signatures clearly seen on Iapetus are consistent the presence of aromatic units of small size (1-10 rings) linked with short aliphatic bridging units similar to the kind envisioned for meteoritic organic complexes (e.g., Kerridge et al. 1987). The aromatic units are thought to be small because the strength of the band indicates the presence of a relatively large number of H-bonding sites per unit $\mathrm{C}$ atom. Because meteoritic insoluble organic matter (IOM) commonly shows a strong aliphatic absorption and little or no aromatic band (e.g., Pendleton et al. 1994), the aromatic units in the Iapetus material maybe significantly smaller than those in the IOM. Note that the absolute strength of the aliphatic band complex (a mixture of $-\mathrm{CH}_{3}$ and $-\mathrm{CH}_{2}$ units) is $\sim 10$ times that of the aromatic band, so the strength of the $3.3-\mu \mathrm{m}$ band on Iapetus indicates that aromatic material is abundant.

The low-albedo material on Iapetus and the other Saturnian satellites has the characteristics of hydrogenated amorphous carbon (HAC) that is similar in composition and 
structure to the carbon-rich organic dust particles in the diffuse interstellar medium (Pendleton \& Allamandola 2002). Interstellar HAC is composed of a mixture of aromatic regions $\left(s p^{2}\right.$ bonded systems with a polycyclic aromatic hydrocarbon (PAH) structure) within a matrix of diamond-like $\left(s p^{3}\right.$ bonded) or polymeric $\left(s p^{3}, s p^{2}\right.$, and $s p^{1}$ bonded) material (Jones 1990). The conditions of formation and subsequent processing by UV, GCR, and charged particles from the magnetosphere of Saturn influence the instantaneous relative abundances of the various structural components as well as the evolution of this material over time.

Mennella et al. (2002) have shown in the laboratory that hydrogenation of carbon particles by $\mathrm{H}$ atoms is the principal mechanism that determines the presence of the 3.4- $\mu \mathrm{m}$ aliphatic $\mathrm{C}-\mathrm{H}$ band in the diffuse interstellar dust. This hydrogenation can occur when small carbon grains are exposed to a flux of $\mathrm{H}$ atoms produced by a microwave discharge. In this process, only the $3.4-\mu \mathrm{m}$ aliphatic $\mathrm{C}-\mathrm{H}$ structure is produced, but when these same particles are heated, the strength of the aliphatic band decreases and the 3.3- $\mu \mathrm{m}$ aromatic band becomes prominent. The aromatic band is most prominent at $T \sim 500 \mathrm{C}$; at higher temperatures dehydrogenation begins to occur and the $\mathrm{C}-\mathrm{H}$ bands rapidly disappear (V. Mennella private communication).

If the low-albedo material on the Saturn satellites originated as interstellar HAC with an intrinsic 3.4- $\mu \mathrm{m}$ aliphatic hydrocarbon band of sufficient strength to allow spectroscopic detection, and if the mechanism of hydration was the same as Mennella et al. (2002) reproduced in the laboratory, then the appearance of the $3.3-\mu \mathrm{m}$ aromatic hydrocarbon band suggests a degree of thermal processing. At the same time, the absence of detectable bands in large regions of the surfaces of the satellites that are otherwise low in albedo suggests the loss of $\mathrm{C}-\mathrm{H}$ bonds through the effects of processing by solar UV, GCR, and charged particles from Saturn's magnetosphere. The dehydrogenation of HAC leaves abundant elemental carbon of graphitic structure and with low albedo and more neutral color than its hydrogenated counterpart. The darkening and dehydrogenation of HAC films by UV irradiation in the laboratory (see Jones 1990 for discussion and references) may represent the same basic mechanism that occurs on planetary surfaces upon prolonged exposure to the space environment, and may be a major factor in accounting for the wide variation in the degree of reddening of solid body surfaces among low-albedo planetary satellites, KBOs, and Centaurs.

The detection of aromatic and aliphatic hydrocarbon bands on Iapetus has implications for the presence of hydrocarbons in the low-albedo material that covers or constitutes the surfaces of a great number of other small bodies in the outer Solar System. The Iapetus material is closely modeled with tholins as the principal refractory component and as the component giving the overall reflected spectral energy distribution (color) over the wavelength range 0.3-4 $\mu \mathrm{m}$ (Owen et al. 2001). Similarly, red-colored KBOs and Centaurs are well modeled with tholins serving the same roles (Barucci et al. 2001, 2005, Cruikshank et al. 1998). At the same time, tholins are known to contain, among other things, both aliphatic and aromatic hydrocarbons (Imanaka et al. 2004, Bernard et al. 2006). It is therefore reasonable assert that the very red-colored surface materials of the wider range of bodies also consist of hydrocarbon-bearing organic complexes, even though the exact structure, composition, and nature are most probably different from the synthetic tholins made in the laboratory.

Naturally occurring organic complexes in meteorites contain aliphatic and aromatic units, as noted above (see also Yabuta et al. 2007), and while the aliphatic component is most prominent in the near-infrared spectra, the insoluble organic matter from some meteorites (C. Alexander, private communication) also shows a prominent aromatic band at $\sim 3.3 \mu \mathrm{m}$. Future investigations will include the modeling of the Iapetus spectrum and 
the spectra of other red-colored bodies in the Solar System using the optical constants derived for meteoritic organic matter. Successful modeling of the spectral shape and albedo levels with meteoritic organics will strengthen the hypothesis that organic solid material is a basic and wide-spread component of the surfaces of many outer Solar System bodies.

\section{Acknowledgements}

I thank Drs. Max Bernstein, Conel Alexander, George Cody, Yvonne Pendleton, Vito Mennella, and Louis Allamandola for many stimulating discussions. The Iapetus spectral information from the VIMS instrument is a part of the NASA-ESA Cassini mission to the Saturn system. I thank my many colleagues on the Cassini mission for their efforts over many years to obtain these and other data.

\section{References}

Barucci, M. A., Cruikshank, D. P., Dotto, E., Ferlin, F., Poulet, F., Dalle Ore, C., Fornasier, S., \& de Bergh, C. 2005, A\&SA (Letter), 439, L1

Barucci, M. A., Doressoundiram, A., \& Cruikshank, D. P. 2004, in: M. Festou, H. Keller, and H. Weaver (eds.), Comets II, (Tucson: Univ. Arizona Press), p. 647

Barucci, M. A., Fulchignoni, M., Birlan, M., Doressoundiram, A., Romon, J., \& Boehnhardt, H. 2001, A\& $A, 371,1150$

Bernard, J-M, Quirico, E., Brissaud, O., Montagnac, G., Reynard, B., McMillan, P., Coll, P., Nguyen, M-J., Raulin, F., \& Schmitt, B. 2006, Icarus, 185, 301

Brown, R. H. et al. 2004, Space Sci. Rev., 115, 111

Brown, R. H. et al. 2006, A\& $A, 446,707$

Buratti, B. J., Cruikshank, D. P., \& the VIMS Team 2005, ApJ (Letters), 622, L149

Chapman, C. R., Morrison, D., \& Zellner, B. 1975, Icarus, 25, 104

Clark, R. N. et al. 2005, Nature, 435, 66

Clark, R. N. et al. 2008, Icarus, 193, 372

Coradini, A. et al. 2008, Icarus, 193, 233

Cruikshank, D. P. \& Dalle Ore, C. M. 2003, Earth, Moon, Planets, 92, 315

Cruikshank, D. P., Imanaka, H., \& Dalle Ore, C. M. 2005, Adv. Space Res., 36, 178

Cruikshank, D. P. \& Morrison, D. 1974, Icarus, 20, 477

Cruikshank, D. P., et al. 1998, Icarus, 135, 389

Cruikshank, D. P., et al. 2007, Nature, 448, 54

Cruikshank, D. P., et al. 2008, Icarus, 193, 344

Degewij, J., Cruikshank, D. P., \& Hartmann, W. K. 1980, Icarus, 44, 541

Gradie, J. \& Veverka, J. 1980, Nature, 283, 840

Hartmann, W. K., Cruikshank, D. P., \& Degewij, J. 1982, Icarus, 52, 377

Imanaka, H. et al. 2004, Icarus,168, 344

Jones, A. P. 1990, MNRAS, 247, 305

Kerridge, J., Chang, S., \& Shipp, R. 1987, Geochim. Cosmochim. Acta, 51, 2527.

Khare, B. N. et al. 1984, Icarus, 60, 127

Mennella, V. et al. 2002, ApJ, 569, 531

MacDonald, G. D., Thompson, W. R., Heinrich, M. et al. 1994, Icarus, 108, 137

Morrison, D. 1973, Icarus, 19, 1

Owen, T. C. et al. 2001, Icarus, 149, 160

Pendleton, Y. J. \& Allamandola, L. J. 2002, ApJS, 138, 75

Pendleton, Y. J., et al. 1994, ApJ, 437, 683

Sagan, C. \& Khare, B. N. 1979, Nature, 277, 102

Yabuta, H. et al. 2007, LPSC XXXVIII, abstract 2304.pdf

Zellner, B., Tholen, D. J., \& Tedesco, E. F. 1985, Icarus, 61, 355 


\section{Discussion}

KWok: If these complex organics, or tholin-like materials, are common in small Solar System bodies, where are they on Earth? I mean, were they destroyed during the thermal processing or by shocks, or during the aggregation process? If they are preserved in smaller bodies, some of them must have been here.

CRuikshank: I presume that this complex material has fallen on Earth and continues to fall on Earth in the form of meteorites and IDPs. The method of emplacement on the Saturn satellite is far from clear. This material is probably quite young, by which I mean maybe no more than a few million years old. They may also be only a few tens of centimeters in thickness, suggesting again that it fell on the surface from some external source, but we don't know where. I am not sure any comments about the Earth are totally relevant to the Saturn system, but surely the stuff has fallen on the Earth from whatever source - comets, most likely - and continues to form both comets and meteorites and asteroids. So I think it's here, it's all around us. It's just has been mixed with the biological materials to the extent that we would never recognize it as an independent component.

MATthews: Dale, the main result of your elegant survey of these deep color materials is that there are tholins deposited on many of these bodies. So what are tholins? Tholins are made, as you pointed out, by supplying energy to a mixture of methane and ammonia or methane and nitrogen. And what we have been saying is that what a tholins is essentially two polymers, polymers of HCN and of acetylene that come from methane and ammonia or methane and nitrogen, so you have hydrocarbon polymers but more importantly, you have the HCN polymers. I am suggesting that the main colored material as you're drawing attention to so importantly is HCN polymers. You know when you polymerize the HCN you are starting with colorless liquid, liquid of HCN whatever, and it gets yellow, orange, red, and then finally black, exactly these colors we're talking about. So I'm really so glad, quite thrilled to have you, making this an important issue, but I am really adding a statement there, a very positive statement, that $\mathrm{HCN}$ polymers are really important. They have the right colors and they are all over the place. They are important molecules because when you hydrolyze HCN polymers you get amino acids, petites, purines, pyrimidines, it is amazing.

Cruikshank: Yes. Well, I appreciate that comment, Cliff, and you are quite right that $\mathrm{HCN}$ is an important component in the laboratory when this material is made in the gas phase and the solid stuff precipitates on the surface of the vessel, there's a residual gas. If we can condense that on a cold finger, we can watch it change in color from a colorless floss to a dark reddish brown glue which is in fact the HCN polymer. So the resulting gas is just the question of the temperature at which the materials is condensed. The residual gas has a lot of HCN which will deposit and polymerize right before your eyes and it is an interesting thing to see. So in the natural setting whatever this tholins is, probably does have an HCN component. Now, why can't we see the nitrile band? We have looked very hard for the nitrile band around 4.6 microns and while there is some sort kind of spectral activity in the data, we cannot identify a nitrile band with any degree of reliability. So we were puzzled by that.

Cessarsky: How did you date the deposits? Where does this age of ten million year come from? 
Cruikshank: The assertion that it is very recent comes from the absence, or the near absence of any bright craters in this black surface. There are about two or three very small craters which apparently have penetrated the surface layer and have spread bright rays of the underlying water ice. Since we think we know something about the cratering frequency, the impact frequency in this part of the Solar System, we can estimate that this number of craters would be produced in something of the order of a million years. There are different models for the cratering rate and some people say it's less than a hundred thousand years and other models suggest maybe as much as ten million years. But that is a good indication of the absence of penetrating impacts that it is a recent deposit. Its thinness, or thickness, is deduced from radar measurements made from Cassini spacecraft which indicated a radar reflectivity, discontinuity, and a depth of a few tens of centimeters. So that's the indication that is a surfacial layer.

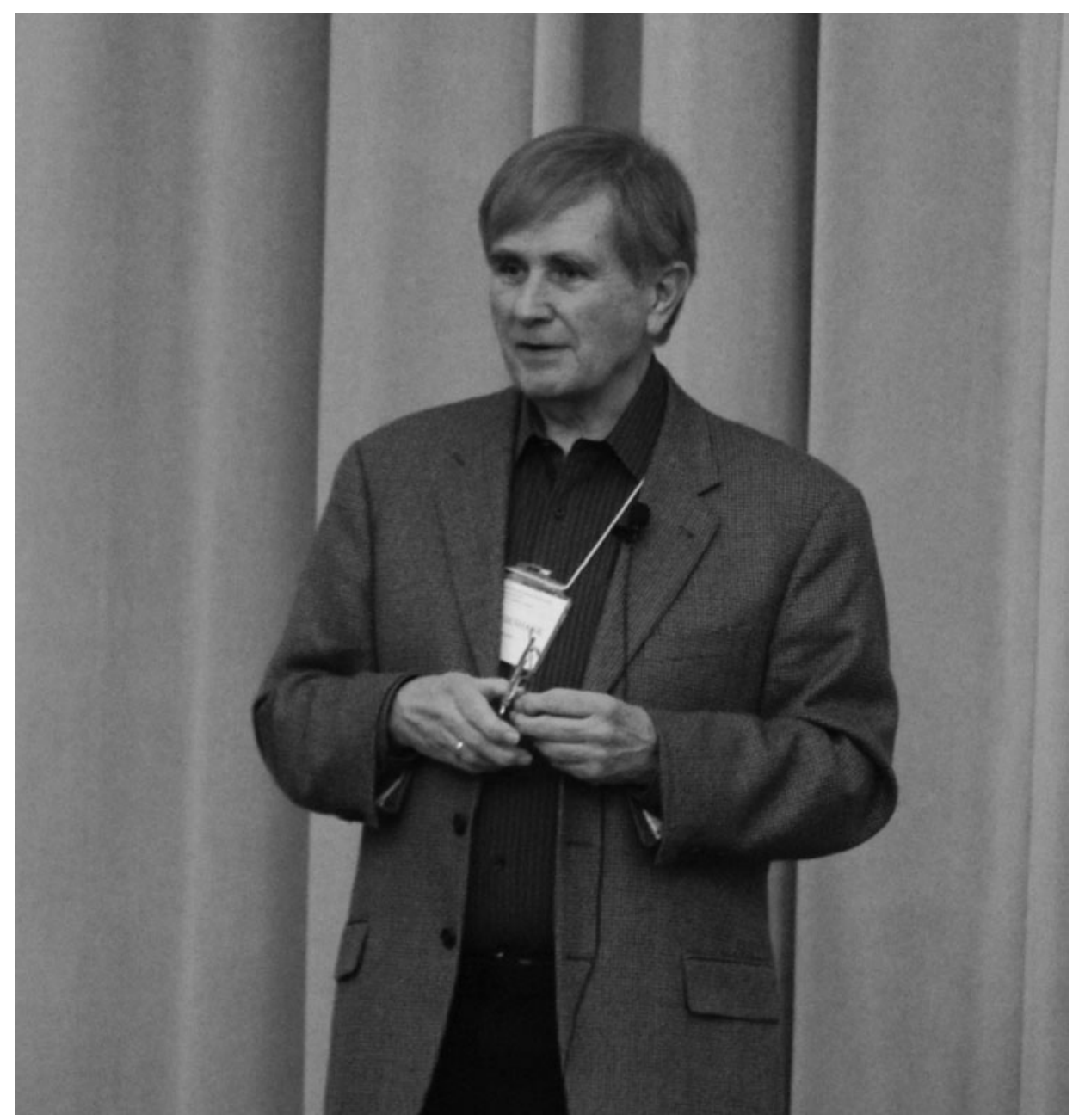

Dale Cruikshank (photo by Sze-Leung Cheung). 